\title{
Effect of folic acid supplementation in pregnancy on gene specific DNA methylation in the child: evidence from a randomised controlled trial
}

\author{
A. Caffrey ${ }^{1}$, H. McNulty ${ }^{1}$, C. Walsh ${ }^{2}$, R. Irwin ${ }^{2}$ and K. Pentieva ${ }^{1}$ \\ ${ }^{1}$ Northern Ireland Centre for Food and Health, Ulster University, Coleraine, BT52 1SA and ${ }^{2}$ Transcriptional \\ Regulation and Epigenetics Group, Ulster University, Coleraine, BT52 1SA.
}

Folic acid is widely recognised for its role in the prevention of neural tube defects and other congenital malformations ${ }^{(1)}$. There is also evidence, although not confirmed, that folic acid supplementation during pregnancy is associated with better cognitive performance and behaviour of the child ${ }^{(2)}$. The underpinning molecular mechanisms for these effects of folate on neurodevelopment are unknown. As folate is an important cofactor in one-carbon metabolism, it is indirectly involved in DNA methylation which in turn is considered to be one of the epigenetic mechanisms controlling gene expression. Observational studies have suggested that folate-rich maternal diets are associated with changes in DNA methylation of the child ${ }^{(3)}$. The current study aims to investigate the effect of folic acid supplementation taken throughout pregnancy on offspring DNA methylation of genes related to brain function.

Samples for the current study were obtained from women participating in a randomised controlled trial which investigated the effect of folic acid supplementation $(400 \mu \mathrm{g} / \mathrm{d})$ during the second and third trimester of pregnancy (FASSTT study). Cord blood samples (n $=86$ ) taken at the time of delivery were assessed for DNA methylation of the following genes associated with brain function: paternally expressed gene 3 (PEG3), insulin-like growth factor 2 (IGF2), brain-derived neurotrophic factor (BDNF), growth factor receptor-bound protein 10 (GRB10), RNA binding motif protein 46 (RBM46) and the retrotransposon long interspersed nuclear element-1 (LINE-1).

\begin{tabular}{lrr}
\hline & \multicolumn{2}{c}{ DNA methylation (\%) } \\
\cline { 2 - 3 } Genes & Placebo $(n$ 45) & Folic Acid $(n$ 41) \\
\hline LINE-1 & $57 \cdot 2(56 \cdot 4-58 \cdot 0)$ & $55 \cdot 9(55 \cdot 1-56 \cdot 8)$ \\
PEG3 & $41 \cdot 1(39 \cdot 9-42 \cdot 2)$ & $41 \cdot 4(40 \cdot 2-42 \cdot 6)$ \\
IGF2 & $51 \cdot 1(49 \cdot 3-52 \cdot 6)$ & $49 \cdot 1(47 \cdot 3-50 \cdot 8)$ \\
GRB10 & $72 \cdot 1(71 \cdot 1-73 \cdot 1)$ & $71 \cdot 4(70 \cdot 4-72 \cdot 5)$ \\
RBM46 & $62 \cdot 8(60 \cdot 6-65 \cdot 1)$ & $0 \cdot 313$ \\
BDNF & $3 \cdot 1(2 \cdot 8-3 \cdot 3)$ & $0 \cdot 067$ \\
\hline
\end{tabular}

Data are expressed as mean $(95 \% \mathrm{CI})$. Differences between groups were analysed by ANCOVA adjusting for birth weight, mother's age and smoking throughout pregnancy.

The results showed significantly lower DNA methylation of LINE-1 and BDNF genes in the cord blood of mothers receiving folic acid compared to those on placebo treatment (Table). Furthermore, significant gender-specific differences between the treatment groups were observed in the DNA methylation of LINE-1, IGF2 and BDNF (data not shown).

These preliminary results indicate that folic acid supplementation $(400 \mu \mathrm{g} / \mathrm{d})$ throughout pregnancy may exert significant effects on cord blood DNA methylation of specific genes related to brain function. Further DNA methylation studies should be conducted to examine other relevant genes and to confirm these results in other populations.

1. Czeizel A \& Dudas I. N Engl (1992) J Med 327, 1832-1835

2. Villamor E, Rifas-Shiman S, Gillman M et al. (2012) Peadiatr Perinat Epid 26, 328-335

3. Haggarty P, Hoad G, Campbell D et al. (2013) Am J Clin Nutr 97, 94-99

For more details please visit: www.bristol.ac.uk/essn 